\title{
Comparisons of Circular Transmit and Linear Receive Compact Polarimetric SAR Features for Oil Slicks Discrimination
}

\author{
Yu Li, ${ }^{1,2}$ Hui Lin, ${ }^{1,2,3}$ Yuanzhi Zhang, ${ }^{2}$ and Jie Chen ${ }^{4}$ \\ ${ }^{1}$ Institute of Space and Earth Information Science, The Chinese University of Hong Kong, Shatin, New Territories, Hong Kong \\ ${ }^{2}$ Shenzhen Research Institute, The Chinese University of Hong Kong, Shenzhen 518057, China \\ ${ }^{3}$ Key Laboratory of Poyang Lake Wetland and Watershed Research, Ministry of Education, Jiangxi Normal University, \\ Nanchang 330022, China \\ ${ }^{4}$ School of Electronics and Information Engineering, Beihang University, Beijing 100037, China
}

Correspondence should be addressed to Hui Lin; huilin@cuhk.edu.hk

Received 26 December 2014; Revised 8 April 2015; Accepted 15 April 2015

Academic Editor: Chengkuo Lee

Copyright (C) $2015 \mathrm{Yu} \mathrm{Li} \mathrm{et} \mathrm{al.} \mathrm{This} \mathrm{is} \mathrm{an} \mathrm{open} \mathrm{access} \mathrm{article} \mathrm{distributed} \mathrm{under} \mathrm{the} \mathrm{Creative} \mathrm{Commons} \mathrm{Attribution} \mathrm{License,} \mathrm{which}$ permits unrestricted use, distribution, and reproduction in any medium, provided the original work is properly cited.

\begin{abstract}
Compact polarimetric (CP) synthetic aperture radar (SAR) has proven its potential in distinguishing oil slicks and look-alikes. Polarimetric information can be retrieved directly from scattering vector or from reconstructed pseudo-Quad-Pol covariance matrix of CP SAR data. In this paper, we analysed features from Circular Transmit and Linear Receive (CTLR) CP SAR data that are derived by taking both of these two methods. $K$-means clustering followed by accuracy assessment was also implemented for performance evaluation. Through experiments that were conducted based on L-band UAVSAR fully polarimetric data, it was found that optimum extraction methods varied for different features. The histogram analysis and segmentation results also demonstrated the comparable performance of CP SAR features in distinguishing different damping properties within oil slicks. This study proposed a framework of statistically analyzing polarimetric SAR (Pol-SAR) features and provided guidelines for determining optimum feature extraction methods from CP SAR data and for marine oil-spills detection and classification.
\end{abstract}

\section{Introduction}

As one of the major marine disasters, oil-spill pollution largely threatens the marine environment. Early warning of oil-spills by remote sensing is very crucial for the pollution evaluation, control, and clean-up operations. Synthetic aperture radar has been widely used in marine applications for its all-day, all-night, and weather-independent capabilities [1]. It has become a cost-effective way to monitor oil pollution in large areas [2]. SAR images of oil slicks have been captured by a variety of SAR sensors, from previous SIR-C, ERS-1/2, ENVISAT, ALOS-1, and RADARSAT-1 to current RADARSAT-2 and TerraSAR-X [3-5]. There are also some operational oil-spill monitoring systems, such as CleanSeaNet satellite monitoring service developed by European Maritime Safety Agency (EMSA), ISTOP (Integrated Satellite Tracking of Pollution) operated by Canadian Ice Service, and semiautomatic SAR oil-spill detection system developed by Kongsberg Satellite Services, Norway [6].
Currently a major difficulty for marine oil slicks detection is the discrimination between mineral oil and its lookalikes (physical or chemical phenomena which cause dark area in SAR images similarly to mineral oil slicks). As compared with single polarimetric SAR, fully polarimetric (FP) SAR has much stronger target classification capability of distinguishing mineral oil and look-alikes [7, 8]. However, FP SAR mode has only half the swath-width due to doubled pulse repetition frequency (PRF) and requires much higher data rate compared with single polarimetric SAR [9]. To overcome this difficulty, compact polarimetric (CP) SAR modes are proposed. CP SAR modes could acquire part of the polarimetric information at reduced PRF and data rate while maintaining the same swath-width as single polarimetric SAR or double the swath-width at the same PRF as fully polarimetric SAR [10]. As a result, CP SAR modes are suitable for the task of broad-area marine surveillance.

Previously there were several studies conducted on $\mathrm{CP}$ SAR based marine applications: Shirvany et al. studied ship 
and oil-spill detection based on degree of polarization derived from dual and CTLR compact polarimetric SAR data [11]. $\mathrm{Xie}$ et al. investigated maritime applications based on $\mathrm{H}$ decomposition on compact and Dual-Pol SAR data [12]. Collins et al. proposed an empirical model to estimate $N$ in variance of incidence angles by fitting the observed data with negative exponent function for the reconstruction of pseudoQuad-Pol SAR data from CP SAR data and used it for ships detection [13]. Li et al. improved Souyris' iterative reconstruction algorithm to derive pseudo-Quad-Pol covariance matrix from CP SAR images of sea surface that is covered by oil slicks [10]. Yin et al. proposed three novel CTLR CP SAR based parameters that are suitable for maritime target detection based on Bragg scattering model and tested their capabilities in oil-spill classification [14]. Salberg et al. utilized the structure of CTLR CP SAR mode to derive features and evaluated their performance in oil-spills detection and suppression of look-alikes [2]. Nunziata et al. proposed and tested several new features that can be derived from CTLR CP SAR data and proved their capabilities in distinguishing slickfree, weak damping slick-covered, and mineral oil-covered sea surfaces [15].

Among all the CP SAR modes, CTLR (also known as hybrid) compact polarimetric SAR mode has the advantages of invariance with the target orientation, stability to the affection of Faraday's rotation [16], and comparable robustness to the affection of systematic errors such as noise and crosstalk [17]. Working in CTLR CP SAR mode, as a small, low mass synthetic aperture radar that flew on the Indian Space Research Organization's Chandrayaan-1 mission, MiniSAR aims to study the crater deposits in the permanently dark areas of the lunar poles from lunar orbit [18]. Currently and in the near future there are and will be several spaceborne SAR sensors that are equipped with this mode, including RADAR Imaging Satellite (RISAT) by India Space Agency, ALOS-2 by Japanese Space Agency (JAXA), SAOCOM of Argentina, and RADARSAT Constellation Missions (RCM) by Canada Space Agency.

In order to take advantage of CP SAR data, generally two strategies have been taken by previous studies. One is to reconstruct pseudo-Quad-Pol SAR matrix from CP SAR data and then conduct Quad-Pol feature analysis [19, 20]. The other is directly extracting features from scattering matrix or stokes vectors of CP SAR data. Recently the latter method is relatively more frequently used $[2,11,14,15]$ while there are also some studies on the improvement of CP SAR reconstruction algorithms for marine applications [10, 13].

The advantage of analyzing CP SAR data based on Quad-pol reconstruction is that all current polarimetric SAR features can then be directly calculated from pseudoQuad-Pol SAR data. However, since CP SAR has insufficient data dimension compared with Quad-Pol SAR mode, the reconstruction algorithms inevitably relay on assumptions of relationships between different polarimetric channels, which may lead to biased estimation. Extracting Pol-SAR features directly from CP SAR features provides another way to utilize CP SAR data, which can avoid errors to be introduced during the process of reconstruction. Since the data of CP SAR modes has different form and physical meanings compared with those in Quad-Pol SAR mode, features extraction algorithms should be adjusted according to their special configuration.

In this paper, we will focus on the evaluation of CP SAR features extracted by using both of these different strategies. Several often used Pol-SAR features that can be derived from both Quad-Pol and CTLR modes will be reviewed and considered in the experiment. Parameters such as normalized difference between the means and relative difference were used to quantitatively analyse statistical behaviour of these Pol-SAR features. Then $K$-means clustering was implemented to demonstrate the performance of oil slicks segmentation, followed by classification accuracy assessment based on overall accuracy and Kappa coefficient.

\section{Polarimetric SAR Features}

In this section, the signal model of fully and CTLR CP SAR mode will be briefly introduced, and then different strategies that take advantage of CP SAR data will be reviewed.

2.1. Basic Representation of SAR Polarimetry. Fully polarimetric SAR systems could obtain the scattering matrix $\mathbf{S}$ of the observed target, which describes the relationship of Jones vectors between the scattered and incident electromagnetic field, in the backscattered coordinate system:

$$
\mathbf{E}^{S}=\frac{e^{-j k r}}{r} \mathbf{S E}^{i},
$$

where $k$ is the wavenumber of the EM wave, $r$ is the distance, and the $2 \times 2$ scattering matrix $\mathbf{S}$ on the traditional linearly horizontal $(h)$ and vertical $(v)$ bases can be described by

$$
\mathbf{S}=\left(\begin{array}{cc}
S_{h h} & S_{h v} \\
S_{v h} & S_{v v}
\end{array}\right)
$$

where the subscript of $S_{i j}$ describes the transmitted and received polarization, respectively.

For the monostatic case, the reciprocity usually holds, which means that the two cross-polarized terms are identical; that is, $S_{h v}=S_{v h}$. And when the reciprocal property is held, the scattering vector $k$ can be defined as

$$
\vec{k}_{3 B}=\left[S_{h h}, \sqrt{2} S_{h v}, S_{v v}\right]^{T} .
$$

Based on $\vec{k}_{3 B}$ the covariance matrix of fully polarimetric SAR data can be derived by

$$
\mathbf{C}=\vec{k}_{3 B} \vec{k}_{3 B}^{* T}=\left[\begin{array}{ccc}
\left|S_{h h}\right|^{2} & \sqrt{2} S_{h h} S_{h v}^{*} & S_{h h} S_{v v}^{*} \\
\sqrt{2} S_{h v} S_{h h}^{*} & 2\left|S_{h v}\right|^{2} & \sqrt{2} S_{h v} S_{v v}^{*} \\
S_{v v} S_{h h}^{*} & \sqrt{2} S_{v v} S_{h v}^{*} & \left|S_{v v}\right|^{2}
\end{array}\right] .
$$

2.2. CTLR Compact Polarimetric SAR Data. In the CTLR CP SAR mode, the radar transmits circularly polarized signal and linearly receives both horizontal and vertical polarizations simultaneously. The $2 \mathrm{D}$ measurement vector $\vec{K}$ is 
the projection of the full backscattering matrix on the transmit polarization state, and in CTLR mode it can be defined as [1]

$$
\vec{K}=\left(\begin{array}{ll}
E_{H} & E_{V}
\end{array}\right)^{T},
$$

where $E_{H}=\left(S_{H H}-j S_{H V}\right) / \sqrt{2}$ and $E_{V}=\left(S_{H V}-j S_{V V}\right) / \sqrt{2}$ and $S_{X Y}$ is the component of the scattering matrix of target, with $X$ denoting the received wave polarization and $Y$ indicating the transmitted wave polarization.
The covariance matrix of compact polarimetric SAR modes, which is derived from their scattering vector, can be defined by [1]

$$
C_{\mathrm{CP}}=2\left\langle\vec{K}_{\mathrm{CP}} \vec{K}_{\mathrm{CP}}^{*}\right\rangle,
$$

where the superscript “*” denotes the transpose conjugate and $\langle A\rangle$ denotes the spatial average of $A$ over a window size, for instance, $5 \times 5$. In this paper, the multilook complex QuadPol SAR data was used to simulate the covariance matrix of CTLR CP SAR mode:

$$
\mathbf{C}_{\mathrm{CTLR}}=\frac{1}{2}\left[\begin{array}{cc}
\left\langle S_{H H}^{2}\right\rangle+\left\langle S_{H V}^{2}\right\rangle+j\left\langle S_{H H} S_{H V}^{*}\right\rangle-j\left\langle S_{H H}^{*} S_{H V}\right\rangle & \left\langle S_{H H}^{*} S_{H V}\right\rangle-j\left\langle S_{H V}^{2}\right\rangle+j\left\langle S_{H H} S_{V V}^{*}\right\rangle+\left\langle S_{H V}^{*} S_{V V}\right\rangle \\
\left\langle S_{H H} S_{H V}^{*}\right\rangle-j\left\langle S_{H V}^{2}\right\rangle+j\left\langle S_{H H}^{*} S_{V V}\right\rangle+\left\langle S_{H V} S_{V V}^{*}\right\rangle & \left\langle S_{H V}^{2}\right\rangle+\left\langle S_{V V}^{2}\right\rangle+j\left\langle S_{H V} S_{V V}^{*}\right\rangle-j\left\langle S_{H V}^{*} S_{V V}\right\rangle
\end{array}\right] .
$$

\subsection{Feature Extraction from Quad-Pol and Reconstructed} Pseudo-Quad-Pol SAR Data. In order to extract Pol-SAR features from CP SAR data through reconstruction of pseudoQuad-Pol data, we employed the iterative algorithm proposed in Dubois-Fernandez et al. [19]. The algorithm is based on the hypotheses that Pol-SAR data of different channels are related and also it assumes that reciprocity and reflection symmetry of geophysical SAR data are held. The algorithm can be basically divided into three steps, namely, initialization, iteration, and reconstruction. The output of CP SAR reconstruction algorithm is pseudo-Quad-Pol covariance matrix. Please note that elements in $C_{\operatorname{Rec}}$ are estimated values, through the reconstruction algorithm:

$$
C_{\mathrm{Rec}}=\left(\begin{array}{ccc}
\left\langle S_{H H} S_{H H}^{*}\right\rangle & 0 & \left\langle S_{H H} S_{V V}^{*}\right\rangle \\
0 & 2\left\langle S_{H V} S_{H V}^{*}\right\rangle & 0 \\
\left\langle S_{V V} S_{H H}^{*}\right\rangle & 0 & \left\langle S_{V V} S_{V V}^{*}\right\rangle
\end{array}\right)
$$

Then the following Pol-SAR features can be extracted from the reconstructed Quad-Pol covariance matrix $C_{\mathrm{Rec}}$ the same as those from Quad-Pol SAR data. The only difference between features extracted from original and reconstructed Quad-Pol covariance matrices is that for the reconstructed Quad-Pol data, multilook cross products between co- and cross-polarimetric channels are assumed to be zero, such as $\left\langle S_{H H} S_{V V}^{*}\right\rangle$. This approximation can be made on scattering coefficients of distributed targets.

(1) $V V^{2}$. The power information of SAR data has been widely used for target detection and classification since the very beginning of marine SAR applications. Generally speaking, $V V^{2}$ is more suitable for oil-spills detection for its high SNR and sensitivity to sea surface roughness while $H V^{2}$ is more suitable for tasks such as ships detection, for its sensitivity to scattered signal from vertical structures. So, in the analysis of this paper, $V V^{2}$ is considered.

(2) Standard Deviation of Copolarized Phase Difference. It was a large breakthrough to realize that phase information within coherence polarimetric SAR data can be used to boost the discrimination between mineral oil and look-alikes. Migliaccio et al. used copolarized phase difference (CPD) to characterize the scattering behavior of oil-spills and biogenic look-alikes [4]. It was discovered that, for sea surface covered by mineral oil, larger CPD standard deviation could be observed while for biogenic slicks a lower CPD standard deviation similar to that of clean sea surface is obtained. From Quad-Pol SAR data, CPD can be derived by

$$
\mathrm{CPD}=\arg \left(S_{H H}\right)-\arg \left(S_{V V}\right)=\arg \left(S_{H H} S_{V V}^{*}\right)
$$

(3) Correlation Coefficient. Correlation coefficient reflects the averaged phase difference between scattering coefficients in $H H$ and $V V$ channels [2]:

$$
\text { Corr }=\frac{\operatorname{Re}\left\langle S_{H H} S_{V V}^{*}\right\rangle}{\sqrt{\left\langle\left|S_{H H}\right|^{2}\right\rangle\left\langle\left|S_{V V}\right|^{2}\right\rangle}}
$$

For clean sea surface where Bragg scattering is dominant, $S_{H H}$ and $S_{V V}$ are highly correlated, so Corr is close to 1, while for mineral oil-covered area where strong damping property is held, $S_{H H}$ and $S_{V V}$ are uncorrelated; then Corr is expected to be much lower.

(4) Conformity Coefficient. Conformity coefficient was used for the study of marine oil slicks detection from CTLR compact polarimetric SAR data [21]. By assuming the reflection symmetry, it can be also derived from Quad-pol SAR data by [21]

$$
\operatorname{Conf} \cong \frac{2\left(\operatorname{Re}\left(S_{H H} S_{V V}^{*}\right)-\left|S_{H V}\right|^{2}\right)}{\left(\left|S_{H H}\right|^{2}+2\left|S_{H V}\right|^{2}+\left|S_{V V}\right|^{2}\right)}
$$

It has been proved that for ocean Bragg scattering, $S_{H V}$ is small, CPD is close to zero, and real part of $S_{H H}$ and $S_{V V}$ cross products is larger than $\left|S_{H V}\right|^{2}$; as a result Conf is positive, 
while for non-Bragg scattering the situation is reverse: Conf is negative.

(5) Coherency Coefficient. The magnitude of the coherency coefficient is useful for oil-spills classification [2]. It can be derived from coherency matrix of Quad-Pol SAR data:

$$
\text { Coh }=\frac{\left|T_{12}\right|}{\sqrt{T_{11} T_{22}}}
$$

where

$$
T=k k^{* T}
$$

and $k$ is the Pauli vector:

$$
k=\frac{1}{\sqrt{2}}\left[\begin{array}{lll}
S_{H H}+S_{V V} & S_{H H}-S_{V V} & 2 S_{H V}
\end{array}\right]^{T} .
$$

(6) Entropy. The polarimetric scattering property of targets can be described by their polarimetric entropy, which is derived from eigenvalue analysis on the coherency matrix [22]. Entropy describes the randomness of the scattering mechanism of ground target. For clean and biogenic slicks covered sea surface, Bragg scattering mechanism is dominant, so low scattering entropy is expected. While for sea surface that is covered by mineral oil, the proportion of volume scattering increases, and the entropy becomes much higher. Polarimetric entropy can be derived from the coherency matrix of Quad-Pol SAR data by [22]

$$
H=\sum_{i=1}^{3}-P_{i} \log _{3} P_{i},
$$

where

$$
P_{i}=\frac{\lambda_{i}}{\sum_{j} \lambda_{i}}
$$

and $\lambda_{i}(i=1,2,3)$ is the eigenvalue of coherency matrix $T$.

2.4. Feature Extraction Directly from CTLR Compact Polarimetric SAR Data. Based on slightly modified definitions and some assumptions, the corresponding Pol-SAR features in Section 2.3 can also be extracted directly from scattering coefficient and coherency matrix of CTLR CP SAR data.

(1) $\left|E_{V}\right|^{2}$ (Corresponding to $V V^{2}$ in Quad-Pol Mode). Due to the Bragg scattering mechanism, in SAR images of sea surface cross-polarized term is usually much larger than copolarized terms, so $E_{H}$ and $E_{V}$ in CTLR mode are comparable to $S_{H H}$ and $S_{V V}$ in Quad-Pol SAR mode. In this study we chose $\left|E_{V}\right|^{2}$ as the corresponding feature to $V V^{2}$.

(2) Standard Deviation of Copolarized Phase Difference $(C P D)$. CPD can be approximately estimated from covariance matrix of CTLR CP SAR data by

$$
\phi_{\mathrm{CPD}}=\arg \left\{-i E_{H} E_{V}^{*}\right\} .
$$

Then its standard deviation within a certain spatial window can be computed.

(3) Correlation Coefficient. Following the same rationale as feature 1, correlation coefficient in CTLR CP SAR mode can be defined as

$$
\text { Corr }=\frac{\operatorname{Re}\left\{-i\left\langle E_{H} E_{V}^{*}\right\rangle\right\}}{\sqrt{\left\langle\left|E_{H}\right|^{2}\right\rangle\left\langle\left|E_{V}\right|^{2}\right\rangle}} .
$$

(4) Conformity Coefficient. Conformity coefficient was originally proposed to distinguish land surface scattering mechanisms [21]. For CTLR CP SAR mode, it is expressed as [21]

$$
\operatorname{Conf} \cong \frac{2 \operatorname{Im}\left(\left\langle E_{H} E_{V}^{*}\right\rangle\right)}{\left\langle E_{H} E_{H}^{*}\right\rangle+\left\langle E_{V} E_{V}^{*}\right\rangle} .
$$

(5) Coherency Coefficient. For CTLR CP SAR mode, the coherency coefficient can be derived by [2]

$$
\operatorname{Coh}=\frac{\left|D_{12}\right|}{\sqrt{D_{11} D_{22}}},
$$

where the coherency matrix $D$ can be defined as

$$
\begin{aligned}
& D \\
& =\left(\begin{array}{cc}
\left\langle E_{H}+i E_{V}\right\rangle^{2} & \left\langle E_{H}+i E_{V}\right\rangle\left\langle E_{H}-i E_{V}\right\rangle^{*} \\
\left\langle E_{H}+i E_{V}\right\rangle^{*}\left\langle E_{H}-i E_{V}\right\rangle & \left\langle E_{H}-i E_{V}\right\rangle^{2}
\end{array}\right) .
\end{aligned}
$$

(6) Entropy. Polarimetric entropy of CP SAR data can be directly calculated by implementing eigenvalue decomposition to the covariance matrix $C_{\mathrm{CP}}$ :

$$
H=\sum_{i=1}^{2}-P_{i} \log _{3} P_{i},
$$

where

$$
P_{i}=\frac{\lambda_{i}}{\sum_{j} \lambda_{i}}
$$

and $\lambda_{i}(i=1,2)$ is the eigenvalue of covariance matrix $C_{\mathrm{CP}}$. Entropy that is derived directly from CP SAR data has similar property with that derived from Quad-Pol SAR data, in describing the complexity of physical scattering mechanisms of targets.

\section{Comparisons of CP SAR Features for Oil Slicks Discrimination}

In this experiment, SAR data acquired by Uninhabited Aerial Vehicle Synthetic Aperture Radar (UAVSAR) is used. It is a reconfigurable, fully polarimetric L-band synthetic aperture radar with a $22-\mathrm{km}$ wide ground swath at $22^{\circ}$ to $65^{\circ}$ incidence 


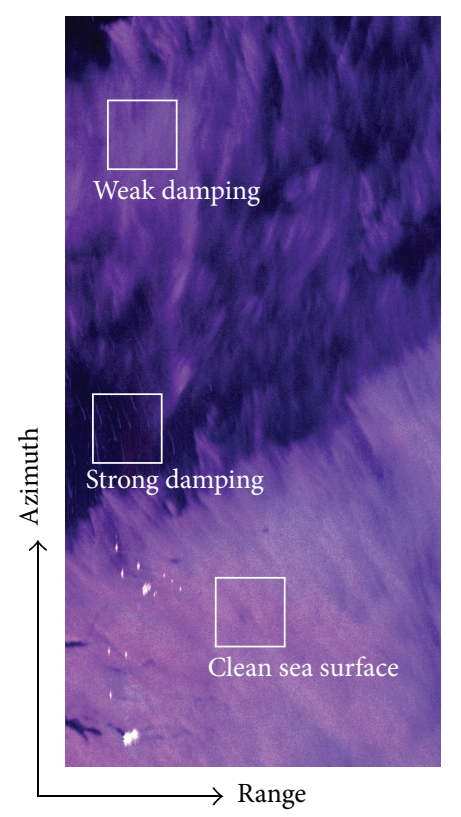

Figure 1: Pseudocolor image of the Quad-Pol SAR data (in dB), with red for $H H^{2}$, green for $H V^{2}$, and blue for $V V^{2}$ (UAVSAR data courtesy NASA/JPL-Caltech).

angles. The experimental image was taken at Gulf of Mexico on September 2, 2009, with flight number 32014-09066, in which an oil slick is present. Multilook complex (MLC) data were used for the analysis, with spatial resolution around $5.5 \mathrm{~m}$. A segment was picked from the image for the convenience of analysis and display. The size of the sample was 1000 pixels in azimuth (vertical) and 500 pixels in range (horizontal) direction. Within the study area, three kinds of typical sea surface are picked out for analysis, namely, strong damping area, weak damping area, and clean sea surface. The pseudocolor image of the studied sample area is shown in Figure 1.

In this study, Pol-SAR features that are derived directly from CP SAR data and via Quad-Pol reconstruction algorithm proposed by Dubois-Fernandez et al. [19], together with those derived from original Quad-Pol SAR data, are compared. Figure 2 shows the flowchart of the whole experiment: MLC data are used as the input in form of covariance matrix. Then, based on the Quad-Pol SAR data, three different ways are used to extract polarimetric SAR features, for discriminating damping status. (1) Pol-SAR features were directly computed from coherence matrix $T$ and covariance matrix C of Quad-Pol SAR data; (2) Quad-Pol reconstruction was implemented on simulated covariance matrix of CTLR compact polarimetric SAR data; then Pol-SAR features were extracted from the reconstructed pseudo-Quad-Pol covariance matrix; (3) CP SAR features were extracted directly from the stokes matrix $\mathbf{g}$, covariance matrix $\mathbf{C}_{\mathrm{CP}}$, and coherence matrix $\mathbf{D}$ of the simulated CTLR CP SAR data without the process of reconstruction. Then all the Pol-SAR features that are derived from different methods were compared and analyzed.
Figure 3 shows the six Pol-SAR features that are derived via different methods. From visual inspection, it can be observed that these corresponding features are very close to each other, except that some noises can be observed from the right part of CP SAR reconstructed features. It was also observed that, in this analyzed case, conformity coefficient holds the strongest capability of distinguishing strong and weak damping sea surface, since it has the largest within-slick contrast. This finding is in correspondence with the analysis results of [23]. Figure 4 is the histograms of these Pol-SAR features. The difference between the distributions of QuadPol SAR features and CP SAR features derived by different methods is generally very small, except that slightly larger difference between CPD derived by different means can be observed.

To statistically analyze the difference between polarimetric characteristics, in distinguishing strong damping, weak damping, and clean sea surface, several statistical differences can be used.

\section{(1) Normalized Distance between Means $\left(d_{\text {norm }}\right)$. Consider}

$$
d_{\text {norm }}=\frac{\left|\mu_{1}-\mu_{2}\right|}{\sigma_{1}+\sigma_{2}}
$$

where $\mu$ and $\sigma$ are mean value and standard deviation, respectively, and subscripts 1 and 2 stand for two different sample areas, for example, weak damping (WD), strong damping (SD), and clean sea surface (sea).

(2) Modified Distance between Samples. Consider

$$
J_{d}=\sum_{i=1}^{C} P_{i}\left[\frac{\left(m_{i}-m\right)^{2}}{\left(\left(1 / N_{i}\right) \sum_{x_{i} \in W_{i}}\left(x_{i}-m_{i}\right)^{2}\right)}\right],
$$

where $C$ are different classes, for example, for oil $i=1$, sea $i=2 ; m_{i}$ is mean value of class $i ; m$ is mean value of all classes; and $P_{i}$ is prior possibility of each class, set to 0.5 in this case.

(3) Bhattacharyya Distance. Consider

$$
B_{d}=-\ln \left(\sum_{i=1}^{n}\left(p_{1}(i), p_{2}(i)\right)^{1 / 2} \times \Delta\right),
$$

where $p_{1}(i)$ and $p_{2}(i)$ stand for possibilities of features from oil and sea sample in $i$ th interval, $n$ stands for the number of intervals used to count possibility function, which is 1000 for this experiment, and $\Delta$ stands for the length of the interval.

Experiments have proved that, in measuring statistical difference between Pol-SAR features of sea surfaces under different damping status, these three measurements have very similar performance. An example is provided in Figure 5, in which three statistical distances represented very similar trend in measuring the statistical distances between weak damping area and clean sea surface. As a result, in this study, we mainly considered the first feature $d_{\text {norm }}$ to keep the analysis concise. $d_{\text {norm }}$ is the statistical distance that is closely related to the performance of minimum distance based classifier. 


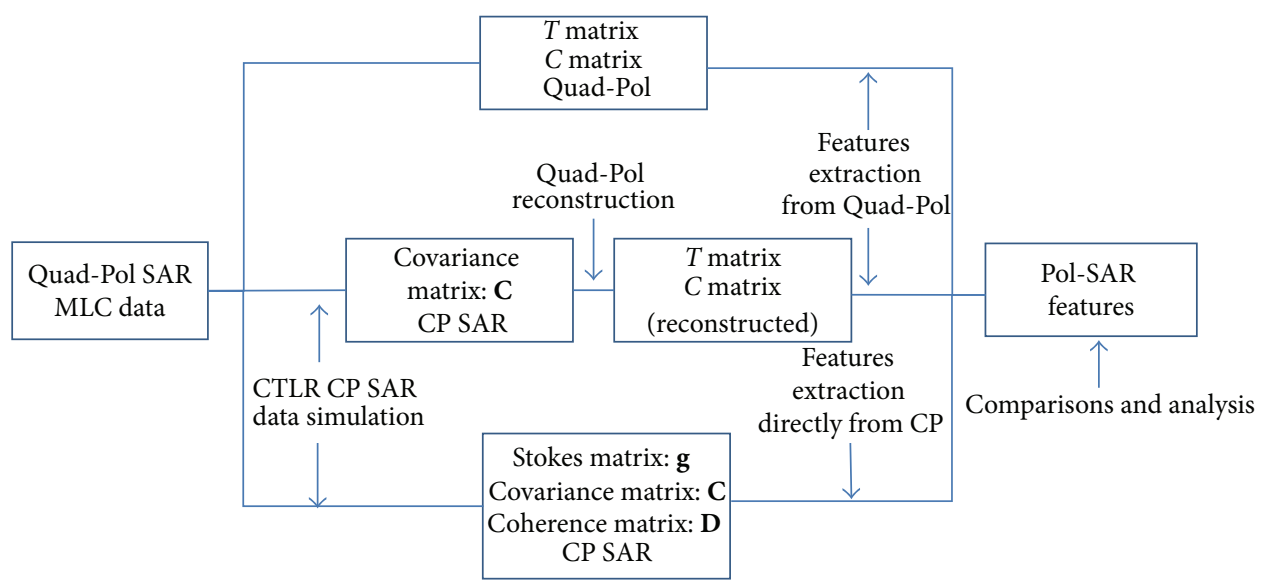

Figure 2: Flowchart of the experiment conducted in this paper.

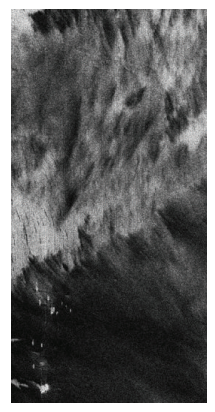

(a1)

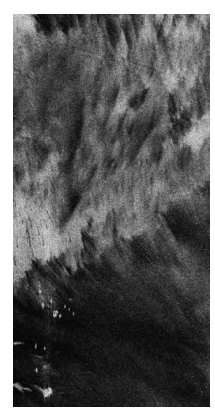

(a2)

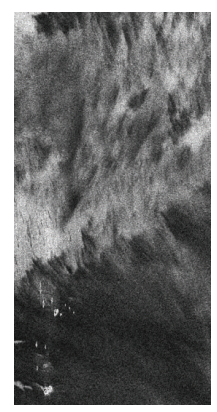

(a3)

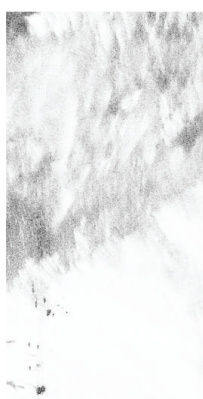

(b1)

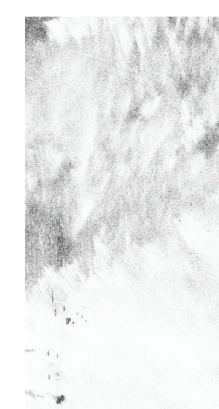

(b2)

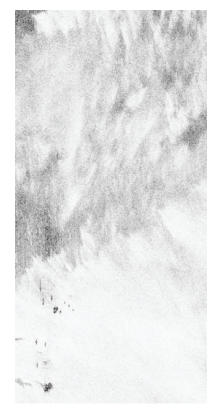

(b3)

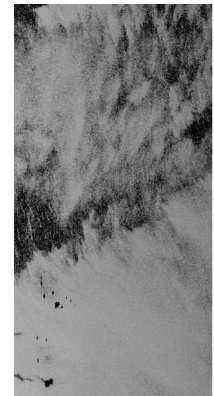

(c1)

Quad-Pol SAR features

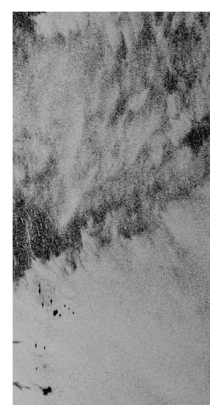

(c2)

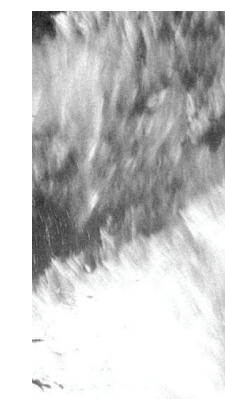

(d2)

CP SAR reconstructed features

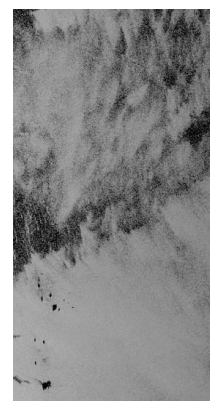

(c3)

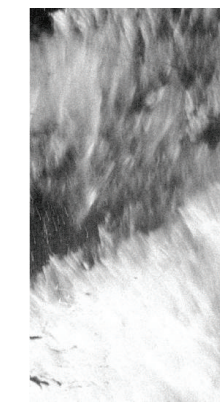

(d3)

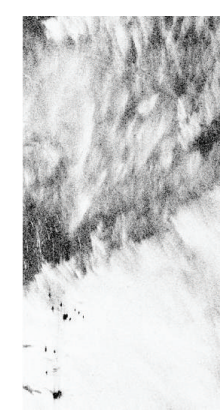

(e1)

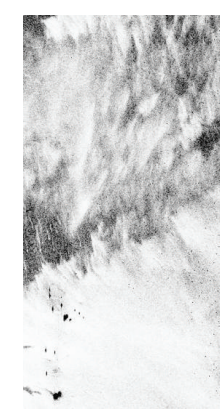

(e2)

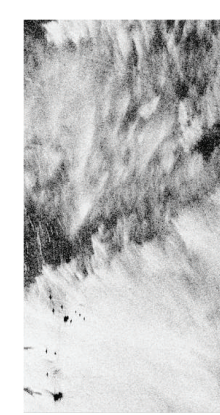

(e3)

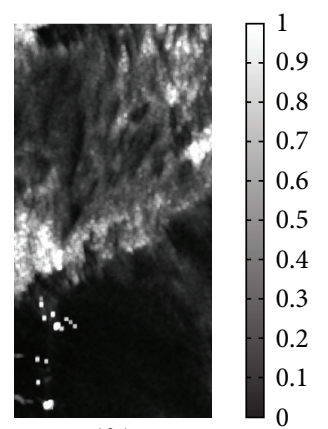

(f1)

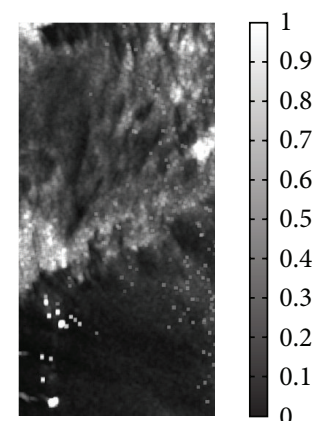

(f2)

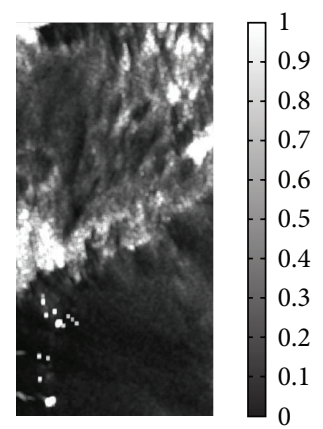

(f3)

CP SAR directly derived features

Figure 3: Pol-SAR features that are derived by using three different methods. From top to bottom (1 3), they are (1) features that are derived from original Quad-Pol SAR data, (2) features that are derived from reconstructed pseudo-Quad-Pol SAR data, and (3) features that are derived directly from CTLR CP SAR mode data. From left to right (a f), they are (a) entropy, (b) coherency coefficient, (c) conformity coefficient, (d) $V V^{2}\left(\left|E_{V}\right|^{2}\right)$, (e) correlation coefficient, and (f) standard deviation of CPD. 

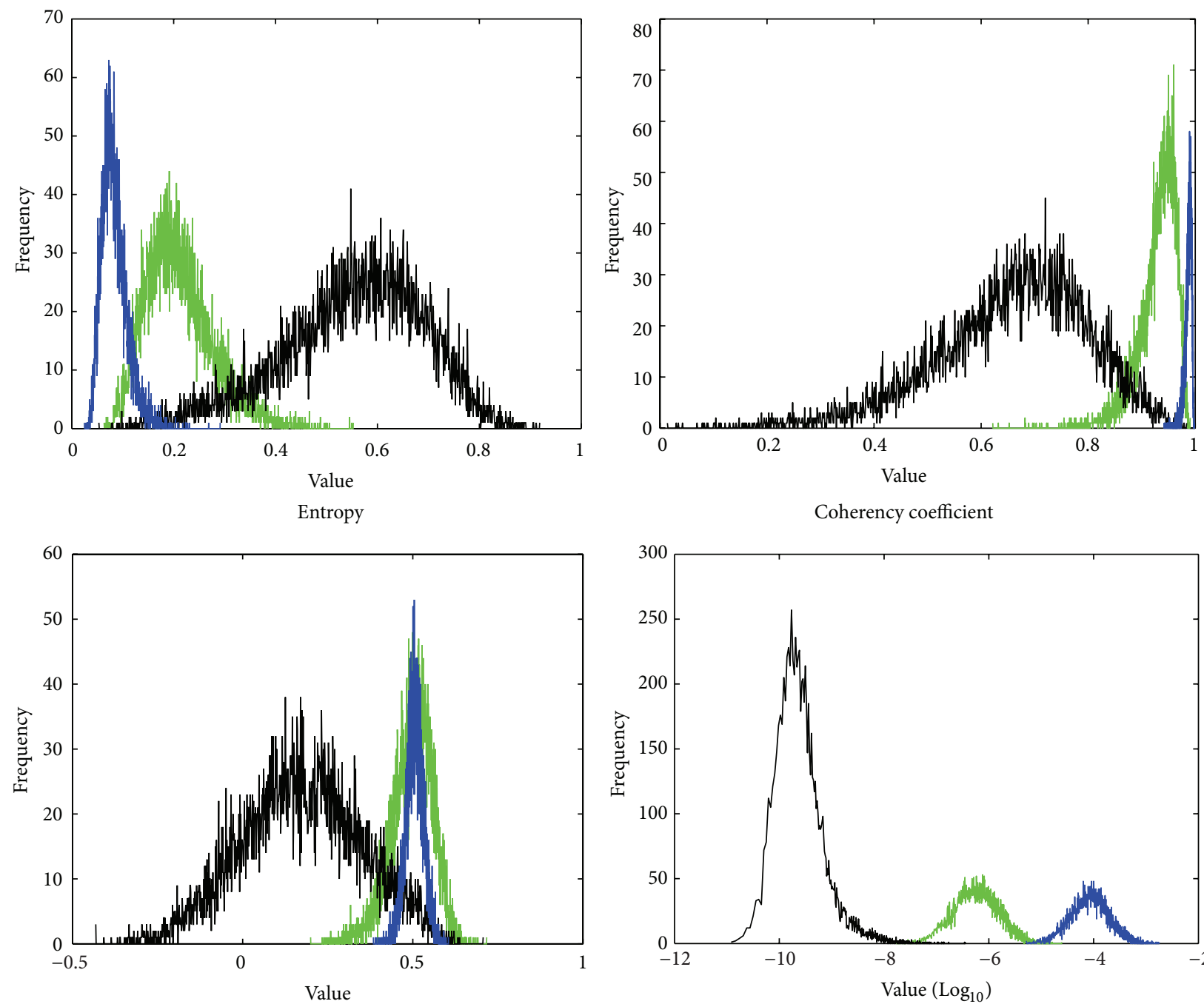

Coherency coefficient

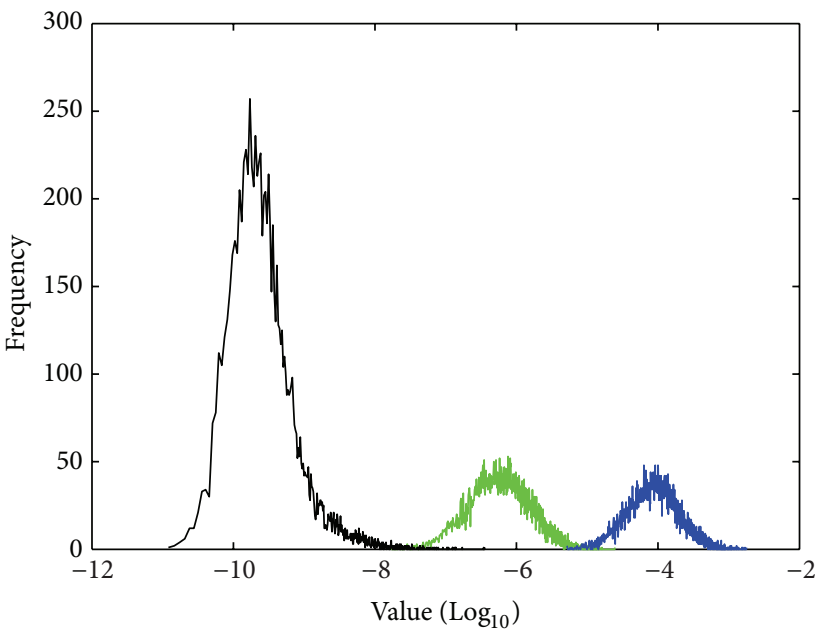

Conformity coefficient
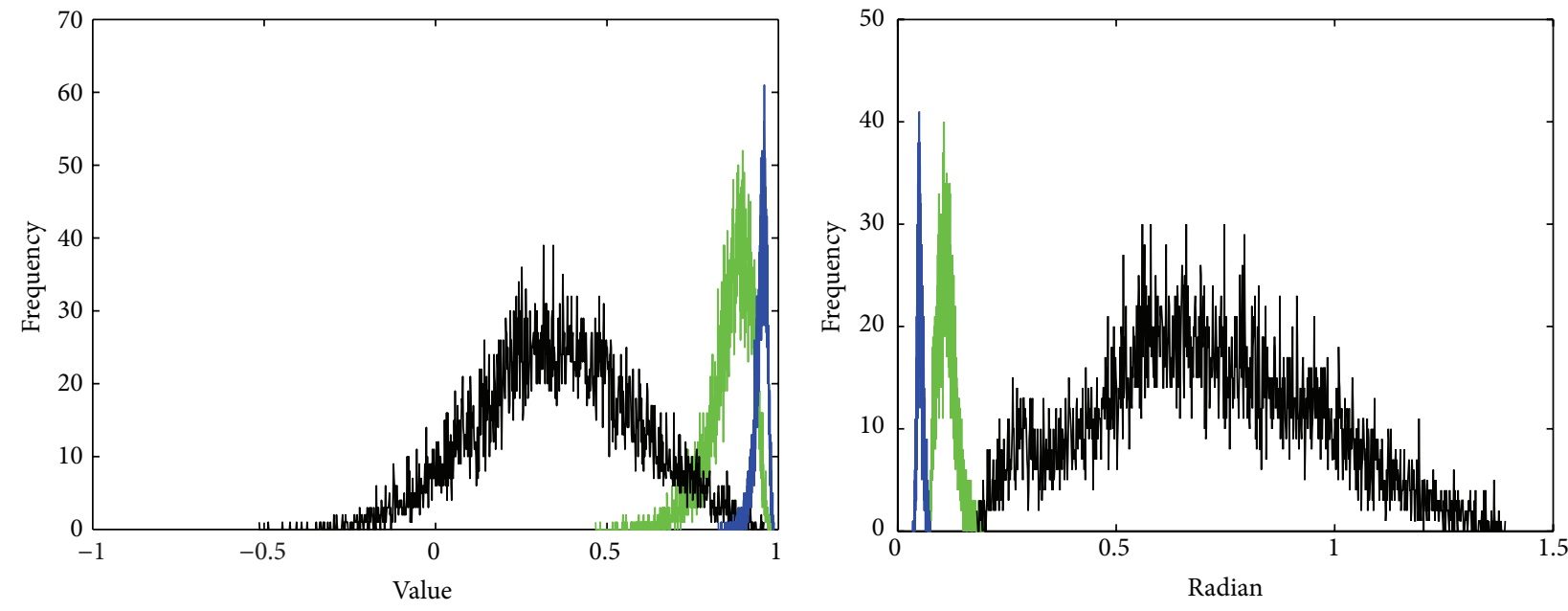

Correlation coefficient

_ Weak damping

Standard deviation of CPD

_ Weak damping
_ Strong damping
Clean sea water

(a) Quad-Pol SAR features

Figure 4: Continued. 

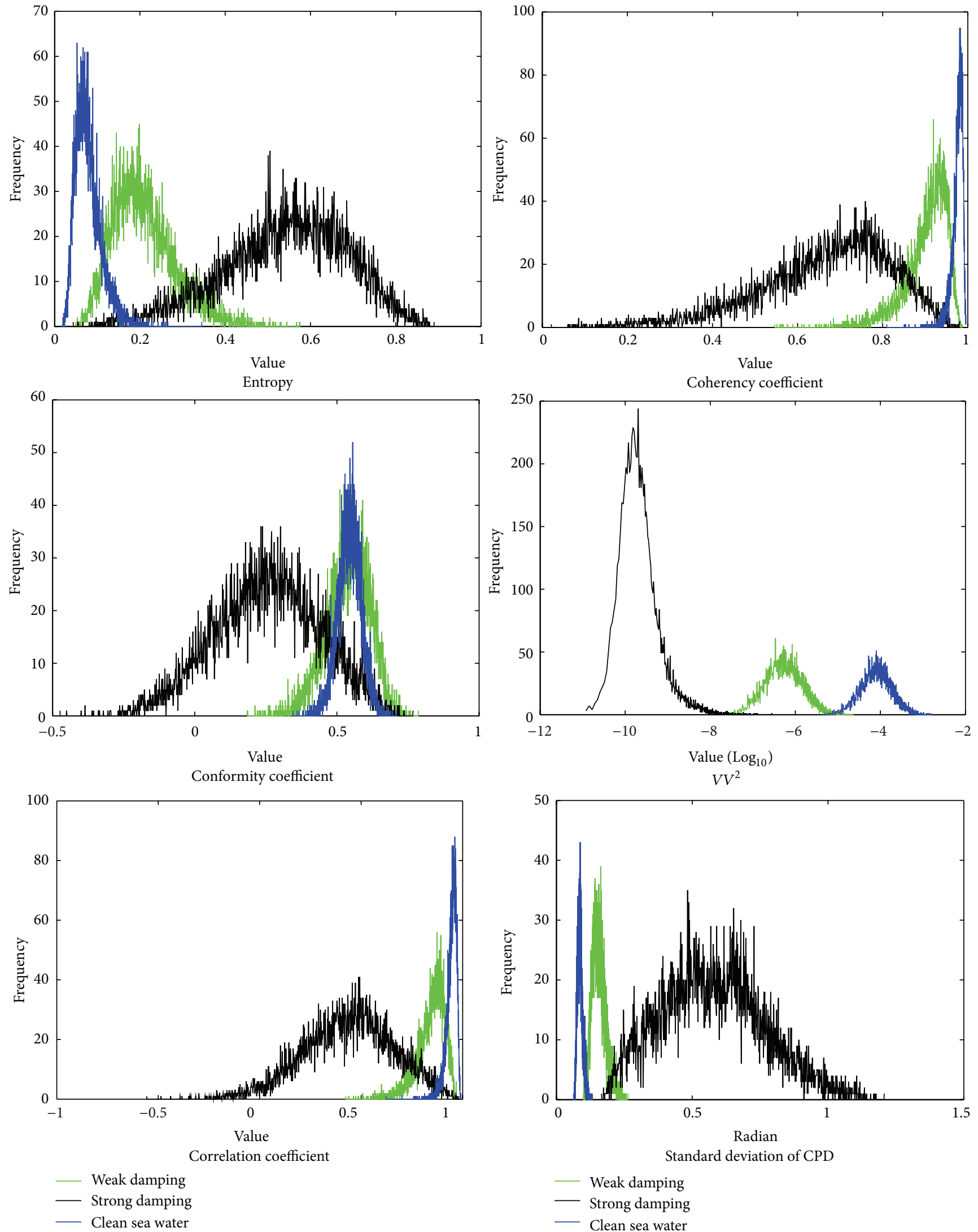

Standard deviation of CPD

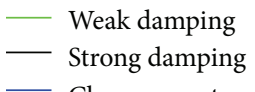

(b) CP SAR reconstructed features

FIgure 4: Continued. 

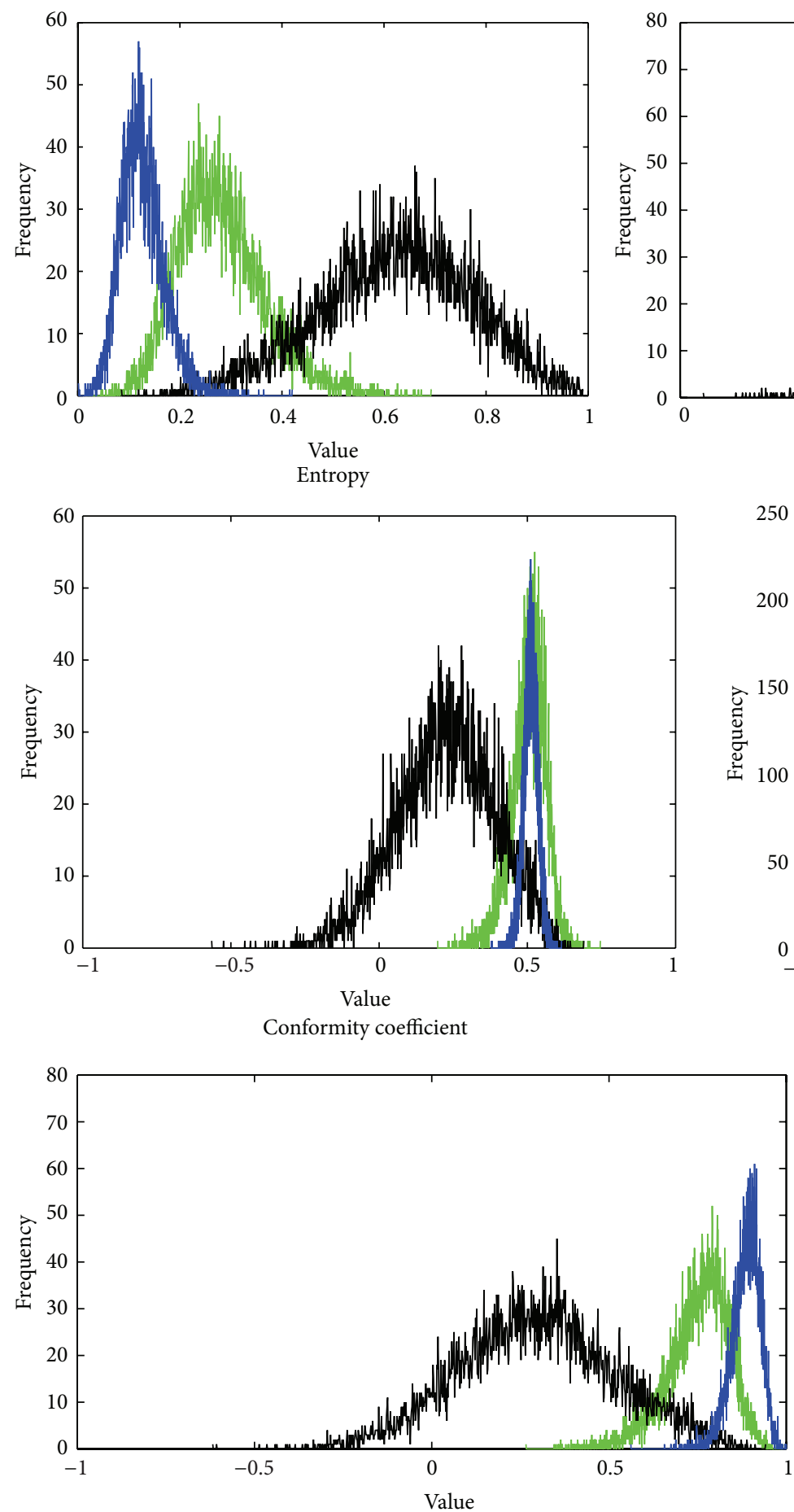

Correlation coefficient

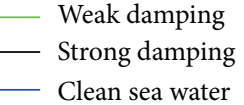

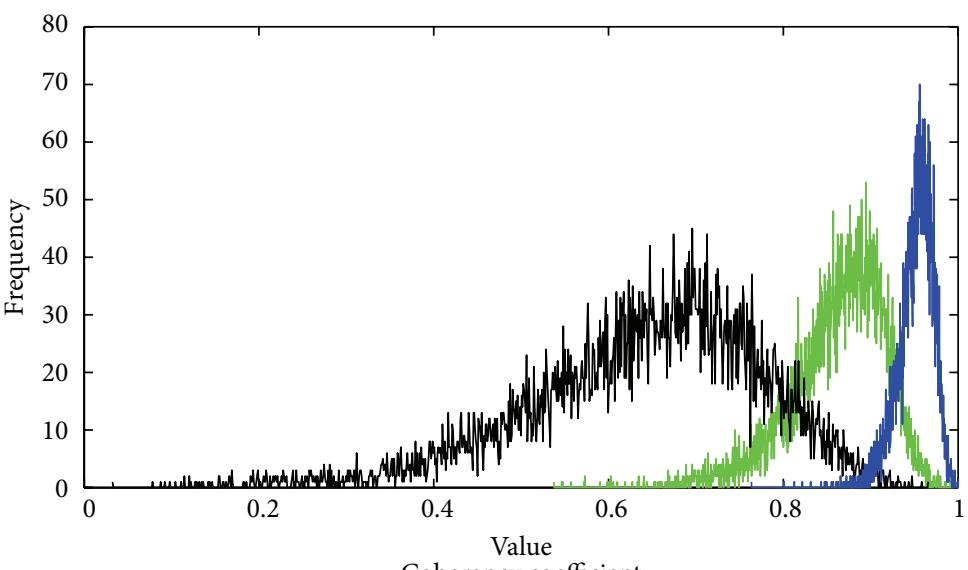

Coherency coefficient
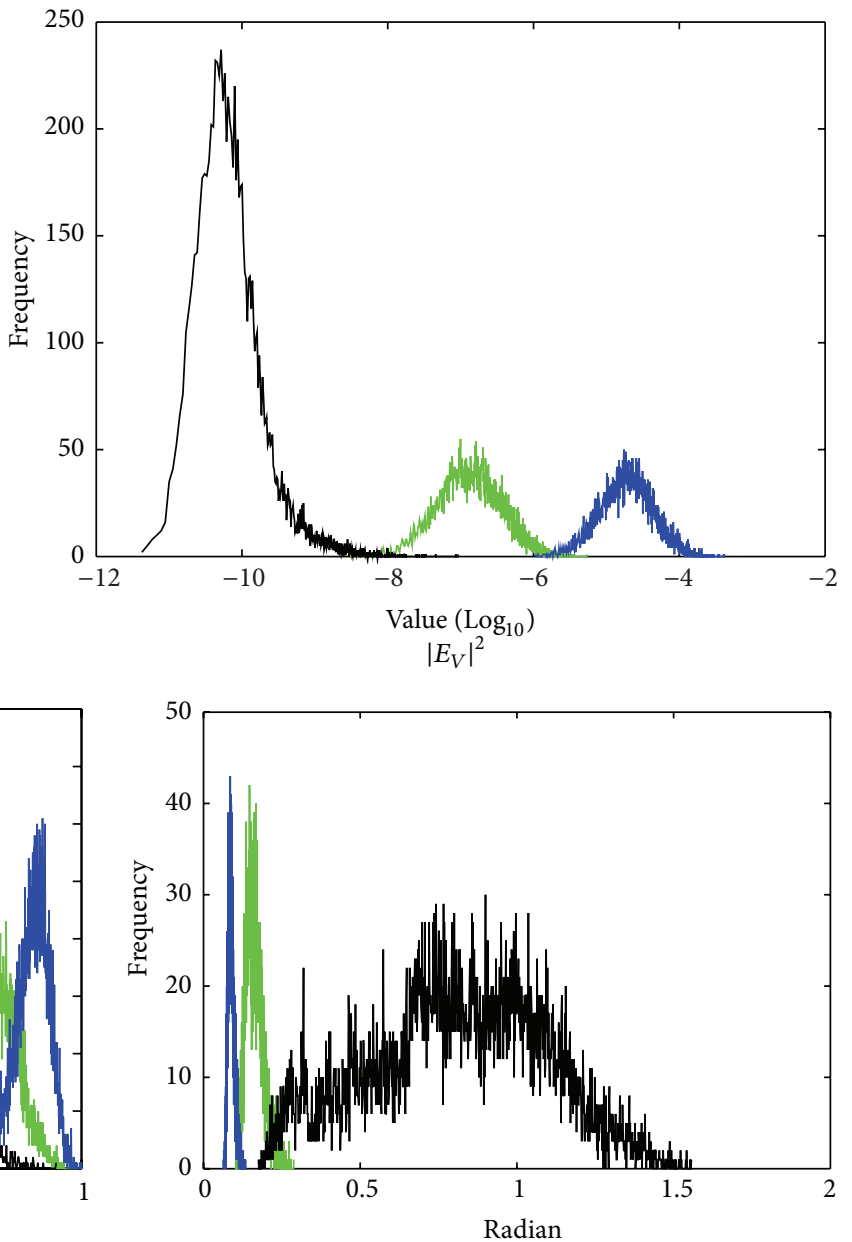

Standard deviation of CPD

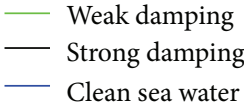

(c) CP SAR directly derived features

Figure 4: Histograms of Pol-SAR features in Figure 3. From top to bottom are (a) features that are derived from Quad-Pol covariance matrix, (b) features that are derived from pseudocovariance matrix that is reconstructed from CP SAR data, and (c) features that are derived directly from simulated CTLR CP SAR scattering matrix, respectively. 


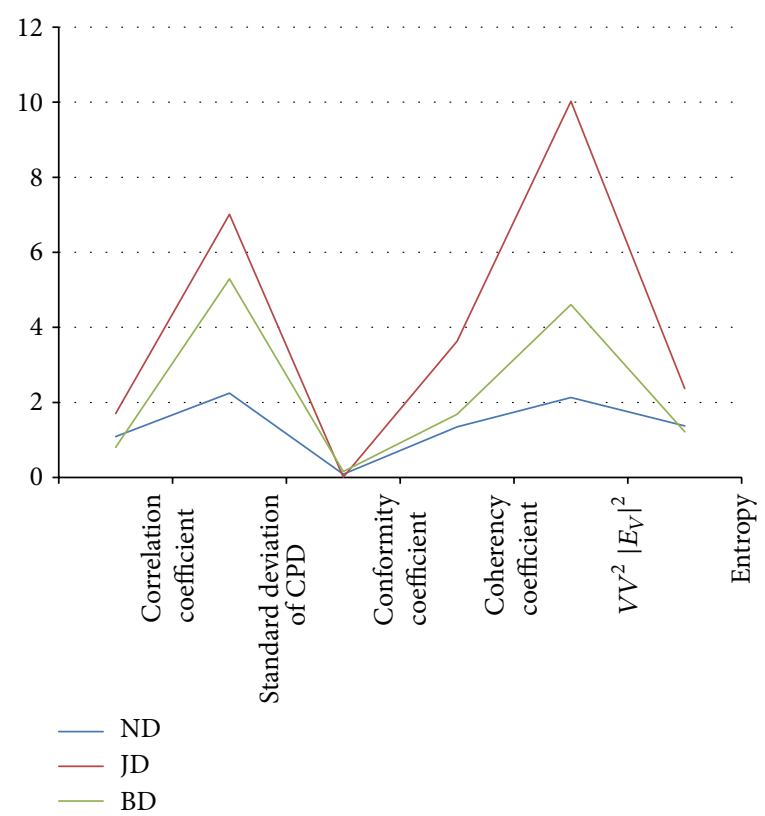

FIgURE 5: Statistical differences of Pol-SAR features between weak damping and clean sea surface measured by different means. ND: normalized distance between means; JD: modified distance between samples; and BD: Bhattacharyya distance.

TABLE 1: $d_{\text {norm }}$ of Pol-SAR features between clean sea surface and weak damping and strong damping areas.

\begin{tabular}{|c|c|c|c|c|c|c|}
\hline \multirow{2}{*}{ Characteristics } & \multicolumn{3}{|c|}{$d_{\text {WD-Sea }}$} & \multicolumn{3}{|c|}{$d_{\text {SD-Sea }}$} \\
\hline & QP & CP Rec. & CP Direct & QP & CP Rec. & CP Direct \\
\hline Corr Co. & 1.0864 & 0.9774 & 1.0459 & 2.4303 & 2.0582 & 2.2730 \\
\hline Std. CPD & 2.2471 & 1.9823 & 1.9761 & 2.4984 & 2.4409 & 2.4394 \\
\hline Conf Co. & 0.0894 & 0.0385 & 0.0954 & 1.6407 & 1.2837 & 1.5191 \\
\hline Coh Co. & 1.3477 & 1.1519 & 1.1924 & 2.2048 & 1.8323 & 2.0127 \\
\hline$V V^{2}\left|E_{V}\right|^{2}$ & 2.1294 & 2.1374 & 2.1124 & 2.7468 & 2.7590 & 2.7330 \\
\hline Entropy & 1.3759 & 1.2513 & 1.2088 & 2.8360 & 2.6281 & 2.5658 \\
\hline
\end{tabular}

Normalized distances calculated from Quad-Pol and CP SAR features are listed in Table 1 . They are calculated by (24) from three sample areas of different damping status. It can be observed that, for all these features, normalized distance between strong damping area and clean sea surface $\left(d_{\text {SD-Sea }}\right)$ is larger than that between weak damping area and clean sea surface $\left(d_{\text {WD-Sea }}\right)$. This is in accordance with physical interpretation: stronger damping properties cause Pol-SAR features in this area to have larger deviation than those in clean sea surface.

From Table 1 it also can be found that, in terms of segmenting oil slicks from oil-free sea background, $V V^{2}$ is the most effective feature. Since the normalized difference of $V V^{2}$ between strong damping area and clean sea surface is the largest, it was one of the most previously used features in traditional single polarimetric SAR based applications. However, in terms of distinguishing weak damping from strong damping properties, other polarimetric SAR features work far better than $V V^{2}$, among them the conformity coefficient works the best, manifesting the fact that for this dataset it has the largest capability in distinguishing different damping status. It should be noted that the feature CPD does not work well on this dataset, but it could be a very good damping status indicator on SAR data of shorter wavelength, for example, $C$ and $X$ bands [4].

Polarimetric SAR features considered in the analysis stand for different physical meanings and they could all help in the oil slicks classification. More analysis and comparisons of these Pol-SAR features in distinguishing different damping status can be found in previous studies [2, 23, 24]. In this paper, however, we mainly focus on analyzing the differences of Pol-SAR features that are derived by two different methods from CP SAR data, namely, directly and through pseudoQuad-Pol reconstruction.

To further evaluate the performance of CP SAR feature extraction methods, relative difference between $d_{\text {norm }}$ that is extracted from Quad-Pol and CP SAR data was calculated:

$$
\mathrm{Rd}=\frac{\left(d_{\text {norm_QP }}-d_{\text {norm_CP }}\right)}{d_{\text {norm_QP }}},
$$

where $d_{\text {norm_QP }}$ stands for $d_{\text {WD-Sea }}$ and $d_{\text {SD-Sea }}$ that are calculated by Quad-Pol data (QP column in Table 1 ) and $d_{\text {norm_CP }}$ stands for those features that are calculated from CP SAR 


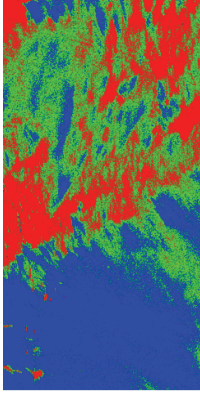

(a1)

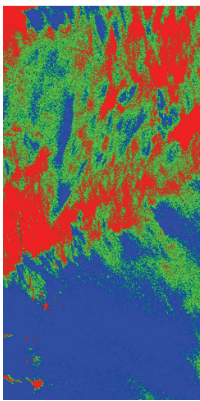

(a2)

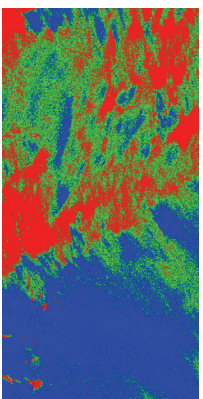

(a3)

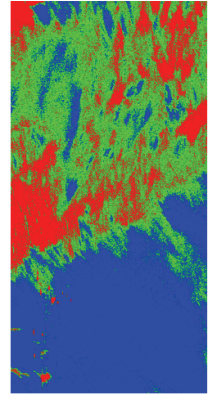

(b1)

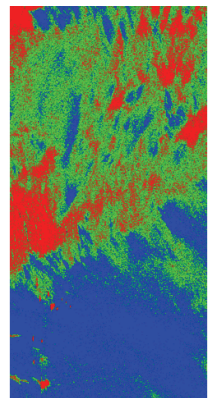

(b2)

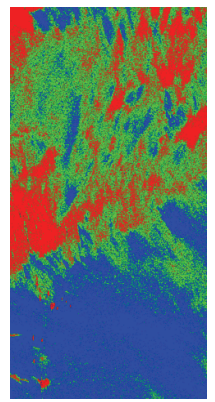

(b3)

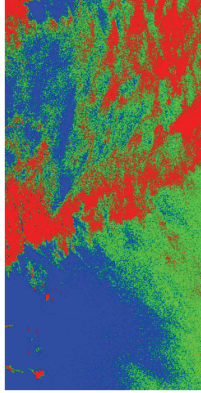

(c1)

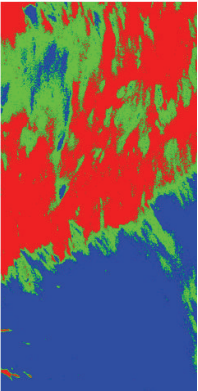

(d1)

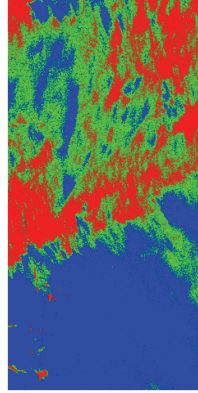

(e1)

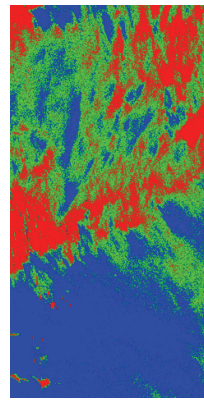

(e2)

(c2)

CP SAR reconstructed features

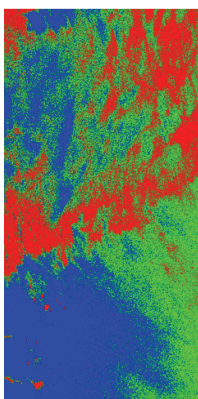

(c3)

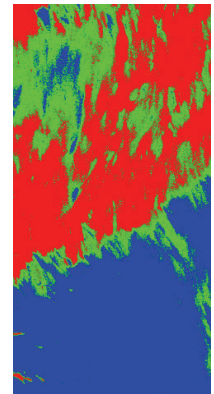

(d2)

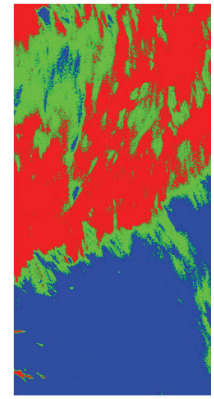

(d3)

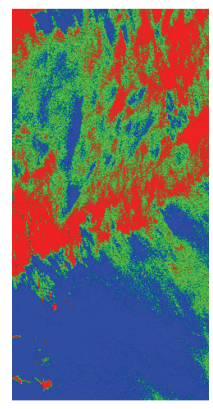

(e3)

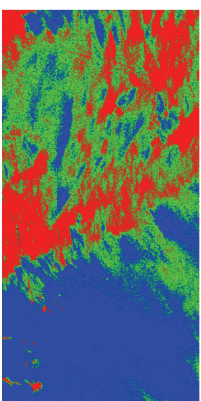

(f1)

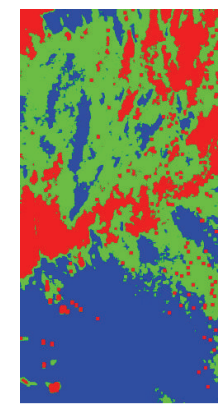

(f2)

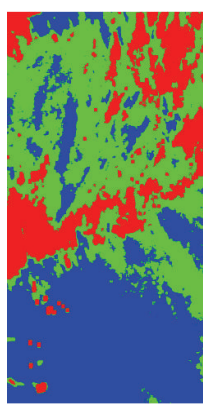

(f3)

CP SAR directly derived features

Figure 6: $K$-means segmentation of Pol-SAR features that are derived by taking three different methods. The results of different features are listed in the same sequence as in Figure 3.

data, either with or without CP SAR reconstruction (CP Rec. or CP Direct column in Table 1).

The smaller the relative difference, the closer the $\mathrm{CP}$ SAR feature to its corresponding original Quad-Pol one and the better the polarimetric information was preserved by taking this method. In Table $2 \mathrm{Rd}_{\mathrm{WD} \text {-sea }}$ stands for the relative distance that is calculated between the normalized difference of Pol-SAR features in weak damping and clean sea surface (column $d_{\text {WD-Sea }}$ in Table 1 ) and similarly for $\mathrm{Rd}_{\mathrm{SD}-\mathrm{sea}}$. From Table 2 it is observed that in calculating features such as $V V^{2}$ $\left(\left|E_{V}\right|^{2}\right)$ and entropy, reconstruction based method resulted in smaller Rd compared with the method that extracts PolSAR features directly. Whereas for features such as correlation coefficient, conformity coefficient, and coherency coefficient, extracting Pol-SAR features directly can achieve lower Rd.
For standard deviation of CPD, performances by taking these two methods are very close.

\section{Experiments on Oil Slicks Segmentation}

In this section, conventional $K$-means clustering was used to partition previously derived Pol-SAR features into three classes. $\mathrm{K}$-means clustering is an unsupervised partitioning method that ensures that members' objects belonging to each class have the smallest distance to its centroid.

Figure 6 shows the results of the $K$-means clustering. The segmentation results are consistent with previous analysis: Conf Co. could reflect the details of damping properties to the largest extent, followed by Corr Co. and other PolSAR features. Based on single polarimetric feature $V V^{2}$, 
TABLE 2: Relative difference Rd between $d_{\text {norm }}$ estimated from Quad-Pol, CP reconstruction, and CP Direct methods.

\begin{tabular}{lcccc}
\hline \multirow{2}{*}{ Features } & \multicolumn{2}{c}{$\mathrm{Rd}_{\text {WD-sea }}$} & \multicolumn{2}{c}{$\mathrm{Rd}_{\text {SD-sea }}$} \\
& QP versus CP Rec. & QP versus CP Direct & QP versus CP Rec. & QP versus CP Direct \\
\hline Corr Co. & -0.10033137 & -0.037279087 & -0.15310867 & -0.06472452 \\
Std. CPD & -0.117840773 & -0.120599884 & -0.023014729 & -0.023602305 \\
Conf Co. & -0.56935123 & 0.067114094 & -0.217590053 & -0.074114707 \\
Coh Co. & -0.145284559 & -0.115233361 & -0.168949565 & -0.087128084 \\
$V V^{2}\left|E_{V}\right|^{2}$ & 0.003756927 & -0.00798347 & 0.004441532 & -0.005024028 \\
Entropy & -0.090558907 & -0.12144778 & -0.073307475 & -0.095275035 \\
\hline
\end{tabular}

TABLE 3: Accuracy assessment of CP SAR features based segmentation results that take Quad-Pol SAR results for ground truth.

\begin{tabular}{lcccc}
\hline \multirow{2}{*}{ Features } & \multicolumn{2}{c}{ Overall accuracy } & \multicolumn{2}{c}{ Kappa coefficient } \\
& CP Rec. & CP Direct & CP Rec. & CP Direct \\
\hline Corr Co. & $84.50 \%$ & $85.401 \%$ & 0.7763 & 0.7613 \\
Std. CPD & $85.17 \%$ & $90.58 \%$ & 0.8207 & 0.8517 \\
Conf Co. & $77.55 \%$ & $92.88 \%$ & 0.6582 & 0.8914 \\
Coh Co. & $82.27 \%$ & $82.78 \%$ & 0.7271 & 0.7329 \\
$V V^{2}\left|E_{V}\right|^{2}$ & $98.63 \%$ & $96.54 \%$ & 0.9791 & 0.9473 \\
Entropy & $88.95 \%$ & $86.29 \%$ & 0.8318 & 0.7917 \\
\hline
\end{tabular}

large homogenous segmentation area was obtained, which manifests its limitation in distinguishing different damping status of oil slicks and other marine phenomena.

Taking the segmentation result derived from Quad-Pol SAR features as ground truth, the CP SAR features based segmentation results can be quantitatively evaluated by overall accuracy and Kappa coefficient. Both of these parameters measure the performance of CP SAR derived features. In Table 3, the results of accuracy assessment are consistent with $\mathrm{Rd}$ listed in Table 2. The highest segmentation accuracy was achieved by $V V^{2}$ derived from pseudo-Quad-Pol reconstruction with overall accuracy of $98.63 \%$ and Kappa coefficient of 0.9791. Among all these features, the segmentation accuracy of conformity coefficient has the largest difference between different CP SAR feature extraction methods. Coincidently in Table 2, Rd of conformity coefficient derived by different methods also holds the largest difference. Although accuracy parameters in Table 3 are computed from the whole image while Rd is only estimated from selected three typical sample areas, they are highly consistent with each other.

\section{Summary}

In this paper, the performance of CTLR compact polarimetric SAR features in distinguishing different damping status of oil slicks is evaluated. Six categories of mainly used Pol-SAR features were considered in the analysis. They were derived from original Quad-Pol SAR data, from reconstructed pseudoQuad-Pol SAR data, and directly from CP SAR mode data, respectively.

To statistically analyze the behavior of these polarimetric SAR features, normalized difference between the means is considered (Table 1). Then relative difference $\mathrm{Rd}$ is computed to measure their capabilities of distinguishing different damping status (Table 2). From the analysis it was found that optimum feature extraction methods from CP SAR varied for different features. Table 2 demonstrated that for $V V^{2}\left(\left|E_{V}\right|^{2}\right)$ and entropy, Souyris' reconstruction algorithm based feature extraction works better. Whereas for correlation coefficient, conformity coefficient, and coherency coefficient, directly extracting Pol-SAR features could maintain the polarimetric information to a larger extent.

Probable explanations for these findings are as follows: polarimetric entropy of Quad-Pol and CP SAR data is calculated based on different numbers of eigenvalues. Besides, $V V^{2}$ and $\left|E_{V}\right|^{2}$ of Quad-Pol and CTLR CP SAR data contain different scattering coefficient elements. As the result, these two features derived from Quad-Pol reconstruction are more similar to those derived from original QuadPol SAR data, for their closer data structure. For features such as correlation coefficient, conformity coefficient, and coherency coefficient, they are closely related to relative phase between polarimetric channels and sensitive to the errors induced during the process of Quad-Pol reconstruction. As the result, extracting those features directly from CP SAR modes may achieve higher accuracy compared with calculating them from reconstructed pseudo-Quad-Pol SAR data, since extracting features directly could avoid adding noise and bias to the sample data.

$K$-means clustering was implemented to all of these Pol-SAR features to examine their capabilities in distinguishing different damping properties. Through classification accuracy assessment based on overall accuracy and Kappa coefficient, the performance of different feature extraction methods can be further compared (see Table 3 ). The result is also consistent with previous analysis result (Table 2 ).

Although the analysis was carried on limited data samples, some basic characteristics of features derived from CP SAR modes can be obtained. CTLR CP SAR mode proved again its potential in obtaining damping status of oil slicks and sea surfaces. The performance of CP SAR features in oil slicks classification is comparable to that obtained in QuadPol SAR modes. Due to some limitations, the analysis in this paper was conducted only on MLC data of UAVSAR, which is processed from SLC data by JPL with a multilook of 12 pixels in azimuth direction and 3 pixels in range direction. It has to be noted that, in the generation of MLC data, symmetry assumption is assumed, and its actual affection on 
reconstruction and feature extraction should be evaluated in future study.

To derive general rules for feature extraction, more data samples under various damping status and sea conditions have to be considered. However this paper proposed a framework of selecting optimum CP SAR features based on statistical analysis on typical data samples. In the near future, there will be plenty of SAR satellites offering compact polarimetric SAR modes, which will provide polarimetric observation of sea surface with larger coverage area. We are confident that this technique will be further developed and make more contribution to operational marine oil-spills monitoring.

\section{Conflict of Interests}

The authors declare that there is no conflict of interests regarding the publication of this paper.

\section{Acknowledgments}

This work is partially supported by the National Key Basic Research Program of China (2015CB954103), the National Natural Science Foundation of China (Grants nos. 41171146 and 41271434), and the Collaborative Innovation Center for Major Ecological Security Issues of Jiangxi Province and Monitoring Implementation (no. JXS-EW-00). The authors would also like to thank JPL, NASA, for providing the UAVSAR data and the anonymous reviewers for their insightful comments that greatly improved the quality of the paper.

\section{References}

[1] M. Gade and W. Alpers, "Using ERS-2 SAR images for routine observation of marine pollution in European coastal waters," Science of the Total Environment, vol. 237-238, pp. 441-448, 1999.

[2] A.-B. Salberg, Ø. Rudjord, and A. H. S. Solberg, "Oil spill detection in hybrid-polarimetric SAR images," IEEE Transactions on Geoscience and Remote Sensing, vol. 52, no. 10, pp. 6521-6533, 2014.

[3] M. Migliaccio, G. Ferrara, A. Gambardella, and F. Nunziata, "A new stochastic model for oil spill observation by means of single-look SAR data," Environmental Research, Engineering and Management, vol. 39, pp. 24-29, 2007.

[4] M. Migliaccio, F. Nunziata, and A. Gambardella, "On the copolarized phase difference for oil spill observation," International Journal of Remote Sensing, vol. 30, no. 6, pp. 1587-1602, 2009.

[5] K. Topouzelis, D. Stathakis, and V. Karathanassi, "Investigation of genetic algorithms contribution to feature selection for oil spill detection," International Journal of Remote Sensing, vol. 30, no. 3, pp. 611-625, 2009.

[6] Y. Zhang, Y. Li, and H. Lin, "Oil-spill pollution remote sensing by synthetic aperture radar," in Advanced Geoscience Remote Sensing, chapter 2, pp. 27-50, 2014.

[7] J. Yang, T. Xiong, and Y.-N. Peng, "Polarimetric SAR image classification by using generalized optimization of polarimetric contrast enhancement," International Journal of Remote Sensing, vol. 27, no. 16, pp. 3413-3424, 2006.
[8] Y. Cui, Y. Yamaguchi, J. Yang, H. Kobayashi, S.-E. Park, and G. Singh, "On complete model-based decomposition of polarimetric sar coherency matrix data," IEEE Transactions on Geoscience and Remote Sensing, vol. 52, no. 4, pp. 1991-2001, 2014.

[9] J. Chen and S. Quegan, "Calibration of spaceborne CTLR compact polarimetric low-frequency SAR using mixed radar calibrators," IEEE Transactions on Geoscience and Remote Sensing, vol. 49, no. 7, pp. 2712-2723, 2011.

[10] Y. Li, Y. Zhang, J. Chen, and H. Zhang, "Improved compact polarimetric SAR Quad-pol reconstruction algorithm for oil spill detection," IEEE Geoscience and Remote Sensing Letters, vol. 11, no. 6, pp. 1139-1142, 2014.

[11] R. Shirvany, M. Chabert, and J.-Y. Tourneret, "Ship and oilspill detection using the degree of polarization in linear and hybrid/compact dual-pol SAR," IEEE Journal of Selected Topics in Applied Earth Observations and Remote Sensing, vol. 5, no. 3, pp. 885-892, 2012.

[12] L. Xie, H. Zhang, C. Wang, F. Wu, B. Zhang, and Y. Tang, "Maritime application using $\mathrm{H}$-a decomposition in compact and dual-pol SAR," in Proceedings of the Asia-Pacific Conference on Synthetic Aperture Radar (APSAR '13), pp. 563-566, September 2013.

[13] M. J. Collins, M. Denbina, and G. Atteia, "On the reconstruction of quad-pol sar data from compact polarimetry data for ocean target detection," IEEE Transactions on Geoscience and Remote Sensing, vol. 51, no. 1, pp. 591-600, 2013.

[14] J. Yin, J. Yang, Z. Zhou, and J. Song, "The extended bragg scattering model-based method for ship and oil-spill observation using compact polarimetric SAR," IEEE Journal of Selected Topics in Applied Earth Observations and Remote Sensing, no. 99, pp. 1-13, 2014.

[15] F. Nunziata, M. Migliaccio, and X. Li, "Sea oil slick observation using hybrid-polarity SAR architecture," IEEE Journal of Oceanic Engineering, vol. PP, no. 99, pp. 1-15, 2014.

[16] J. Chen and S. Quegan, "Calibration of spaceborne CTLR compact polarimetric low-frequency SAR using mixed radar calibrators," IEEE Transactions on Geoscience and Remote Sensing, vol. 49, no. 7, pp. 2712-2723, 2011.

[17] R. K. Raney, "Hybrid-polarity SAR architecture," IEEE Transactions on Geoscience and Remote Sensing, vol. 45, no. 11, pp. 3397-3404, 2007.

[18] R. K. Raney, P. D. Spudis, B. Bussey et al., "The lunar miniRF radars: hybrid polarimetric architecture and initial results," Proceedings of the IEEE, vol. 99, no. 5, pp. 808-823, 2011.

[19] P. C. Dubois-Fernandez, J.-C. Souyris, S. Angelliaume, and F. Garestier, "The compact polarimetry alternative for spaceborne SAR at low frequency," IEEE Transactions on Geoscience and Remote Sensing, vol. 46, no. 10, pp. 3208-3222, 2008.

[20] M. E. Nord, T. L. Ainsworth, J.-S. Lee, and N. J. S. Stacy, "Comparison of compact polarimetric synthetic aperture radar modes," IEEE Transactions on Geoscience and Remote Sensing, vol. 47, no. 1, pp. 174-188, 2009.

[21] B. Zhang, W. Perrie, X. Li, and W. G. Pichel, "Mapping sea surface oil slicks using RADARSAT-2 quad-polarization SAR image," Geophysical Research Letters, vol. 38, Article ID L10602, 2011.

[22] S. R. Cloude and E. Pottier, "An entropy based classification scheme for land applications of polarimetric SAR," IEEE Transactions on Geoscience and Remote Sensing, vol. 35, no. 1, pp. 6878, 1997. 
[23] Y. Li, H. Lin, Y. Zhang, and J. Chen, "Analysis of polarimetric features from CTLR compact polarimetric SAR data for discriminating oil slick damping status," in Proceedings of the IEEE International Geoscience and Remote Sensing Symposium, pp. 3490-3493, July 2014.

[24] S. Skrunes, C. Brekke, and T. Eltoft, "Characterization of marine surface slicks by radarsat-2 multipolarization features," IEEE Transactions on Geoscience and Remote Sensing, vol. 52, no. 9, pp. 5302-5319, 2014. 

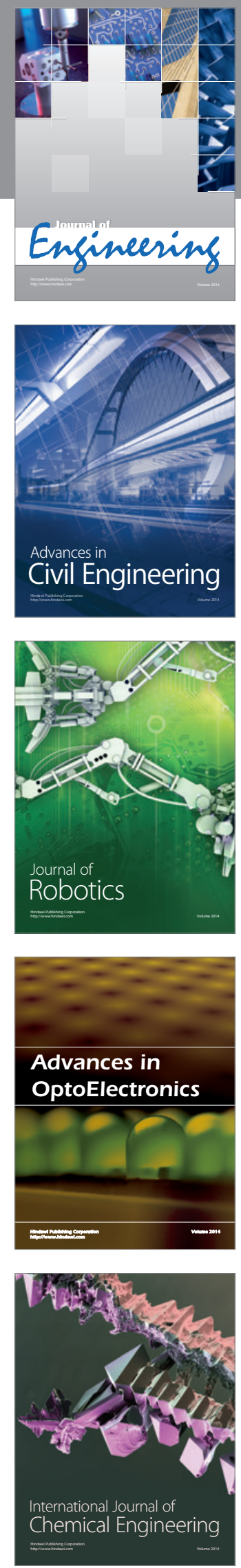

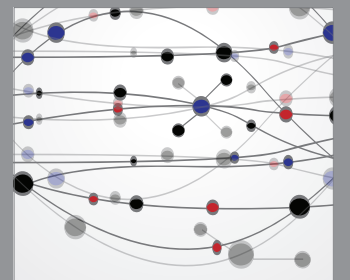

The Scientific World Journal
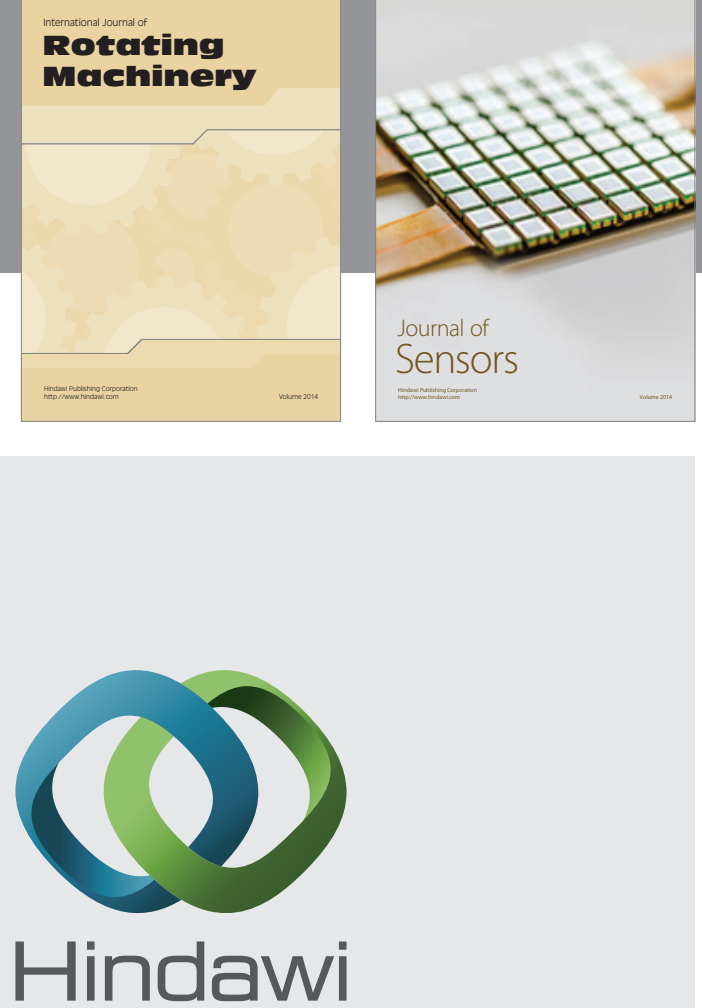

Submit your manuscripts at http://www.hindawi.com
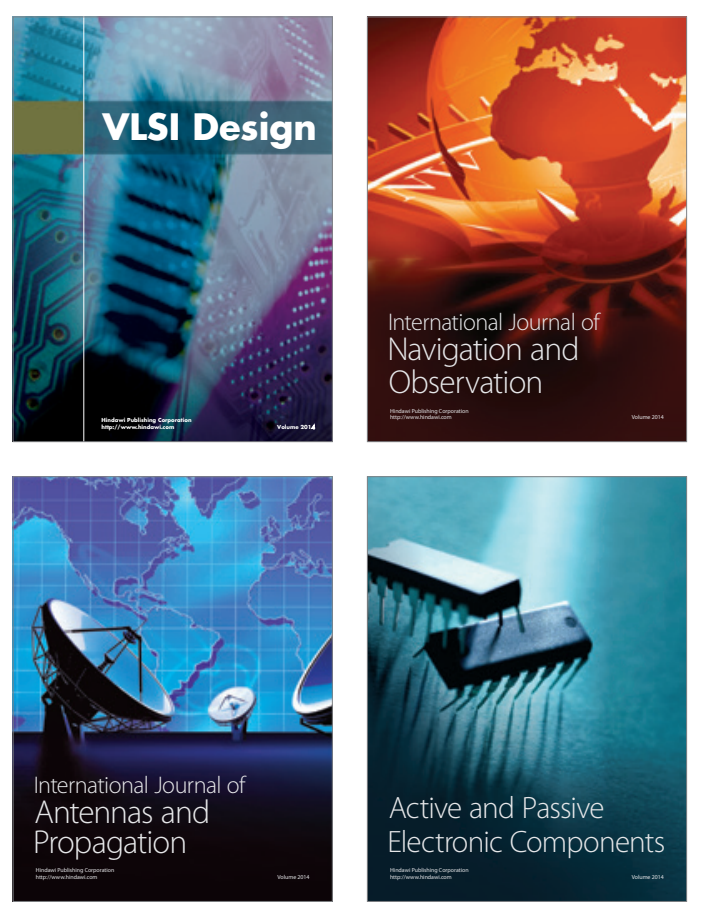
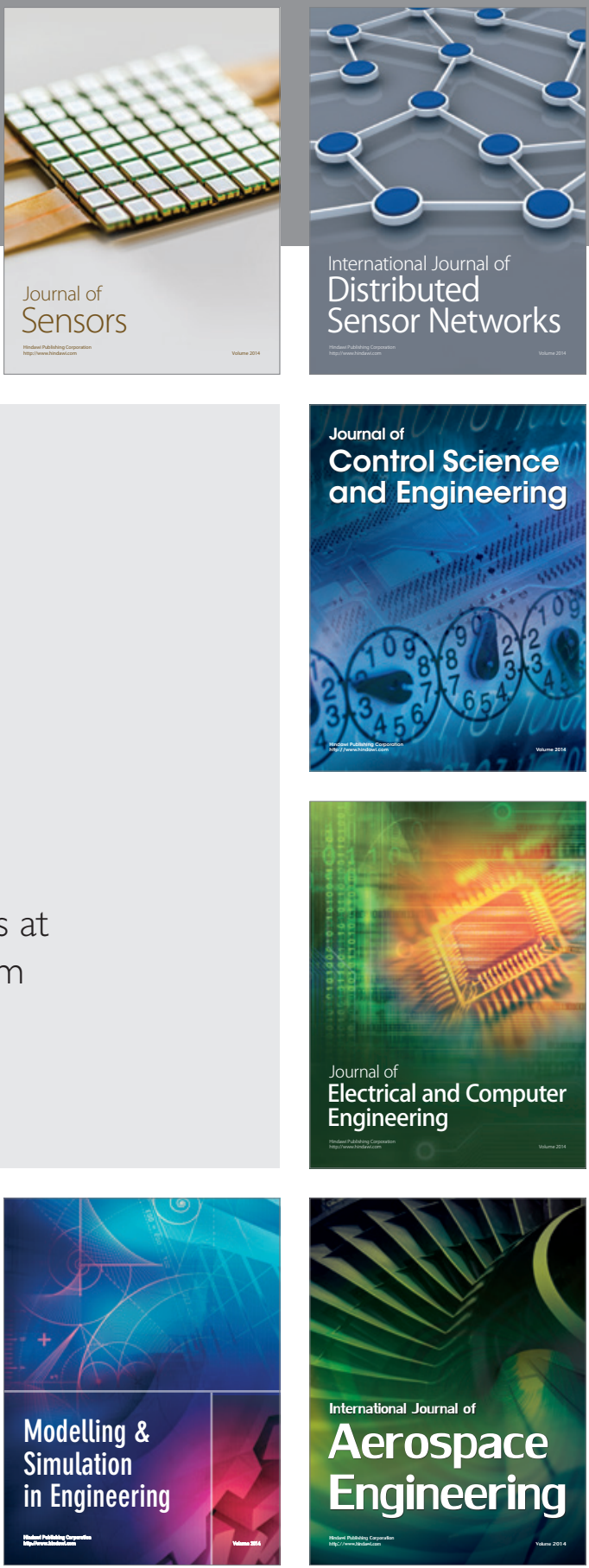

Journal of

Control Science

and Engineering
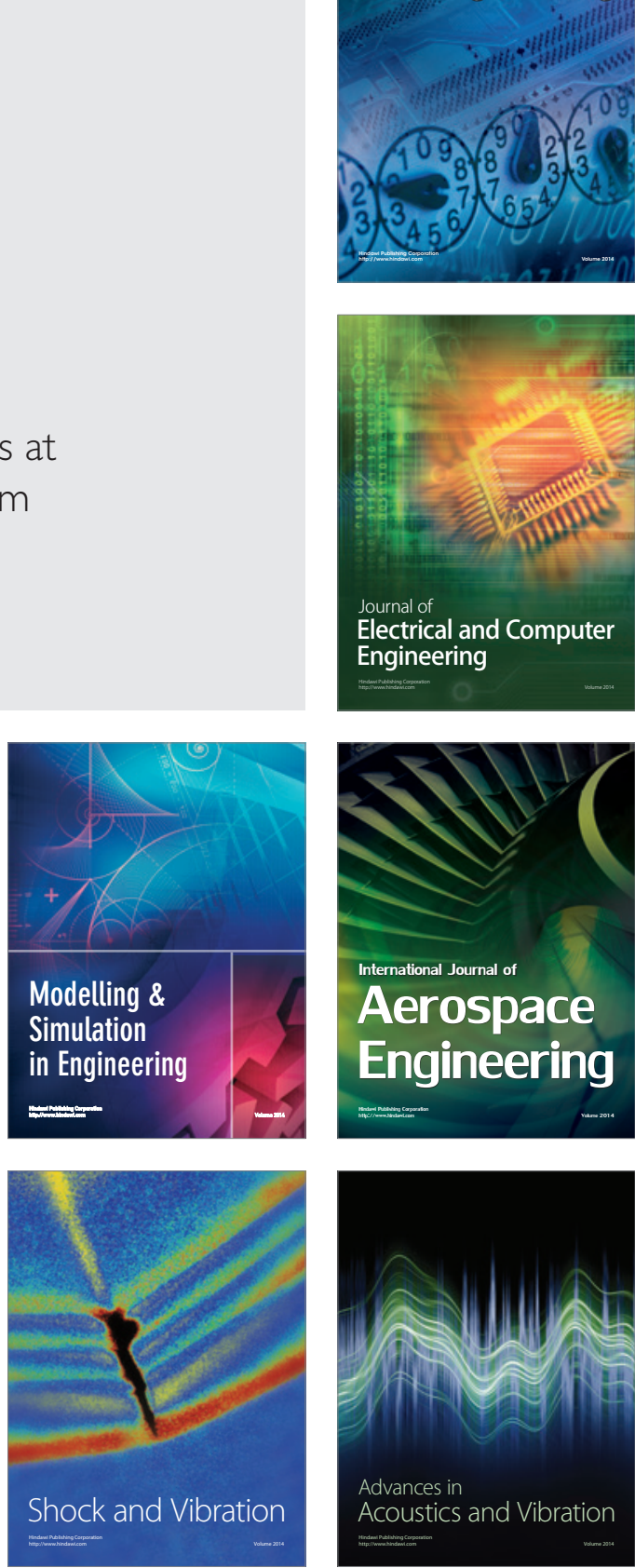